\title{
Klumpke Palsy
}

National Cancer Institute

\section{Source}

National Cancer Institute. Klumpke Palsy. NCI Thesaurus. Code C116724.

Weakness or paralysis of muscles in the forearm or hand due to damage of the lower brachial plexus. 